\title{
Data cleaning for image-based profiling
}

\section{enhancement}

\author{
Arghavan Rezvani ${ }^{1 \pi}$, Mahtab Bigverdi ${ }^{1 \top}$, Mohammad Hossein Rohban ${ }^{1 *}$ \\ ${ }^{1}$ Department of Computer Engineering, Sharif University of Technology, Tehran, Tehran, Iran
}

* Corresponding author

Email : $\underline{\text { rohban@sharif.edu }}$

${ }^{\top}$ These authors contributed equally to this work. 


\section{Abstract}

With the advent of high-throughput assays, a large number of biological experiments can be carried out. Image-based assays are among the most accessible and inexpensive technologies for this purpose. Indeed, these assays have proved to be effective in characterizing unknown functions of genes and small molecules. Image analysis pipelines have a pivotal role in translating raw images that are captured in such assays into useful and compact representation, also known as measurements. CellProfiler is a popular and commonly used tool for this purpose through providing readily available modules for the cell/nuclei segmentation, and making various measurements, or features, for each cell/nuclei. Single cell features are then aggregated for each treatment replica to form treatment "profiles." However, there may be several sources of error in the CellProfiler quantification pipeline that affects the downstream analysis that is performed on the profiles. In this work, we examined various preprocessing approaches to improve the profiles. We consider identification of drug mechanisms of action as the downstream task to evaluate such preprocessing approaches. Our enhancement steps mainly consist of data cleaning, cell level outlier detection, toxic drug detection, and regressing out the cell area from all other features, as many of them are widely affected by the cell area. We also examined unsupervised and weakly-supervised deep learning based methods to reduce the feature dimensionality, and finally suggest possible avenues for future research.

Key terms: high throughput microscopy; image-based profiling; data cleaning; outlier detection; deep learning

\section{Introduction}

High-throughput image-based assays have proved to be an effective predictive tool in early stages of drug discovery through automated microscopy and image analysis, which make quantification of cellular 
morphological responses possible at a large scale (1). These experiments often involve growing cells in multi-well plates, and then treating cells in each well with a small molecule, or genetic perturbation. Image-based profiling has diverse and powerful applications, which include identification of gene and allele functions and targets, and mechanisms of action (MoA) of drugs (2). Prediction of drug MoAs through such assays potentially saves drug discovery process costs when applied early on.

The typical workflow in analysis of images that are produced by these assays include illumination correction, nuclei/cell segmentation, quality control, morphological feature measurement, batch effect removal, data normalization, feature selection/dimensionality reduction, and finally aggregation of single cell measurements into image-based profiles per well (2). The initial steps such as the illumination correction, segmentation, and feature extraction are not investigated in this work. We rather mainly focus on approaches that preprocess the features that are extracted by CellProfiler, an open-source software that aims at automating most of these steps. We conduct a comprehensive study on how such techniques could potentially improve the profile's quality.

Data cleaning is a key step for enhancing image-based profiling. A major step in image-based profiling and data cleaning is cell-level quality control. Outlier cells, which are not showing any valid biological effect, may result from errors in different parts of the pipeline. For instance, an error in the segmentation step may result in overly small or large cells, and bias the profiles heavily as a result. Detecting and removing outlier cells can highly improve the profile quality. We propose to use an unsupervised outlier detection method in this work, and evaluate its effectiveness in the context of MoA prediction. More specifically, histogram-based outlier detection is applied on each plate individually, and by removing the single-cells detected as outlier by this method, the final results are improved.

In addition, we found that the domain specific feature preprocessing, as a data cleaning step, could enhance the relevant downstream task. In summary, we found that the cell area is a key contributor to many 
of the features, and hence the similarity metric is highly influenced by it. Therefore, we propose to neutralize the effect of cell area on the other features to be able to capture more meaningful information in the profiles. Major steps of this work are described in Fig. 1. Finally, we would investigate various general purpose methods for dimensionality reduction and representation learning from the classical features that are extracted by CellProfiler, and found that, as opposed to the domain specific preprocessing step, they are not effective in improving the prediction ability of profiles.

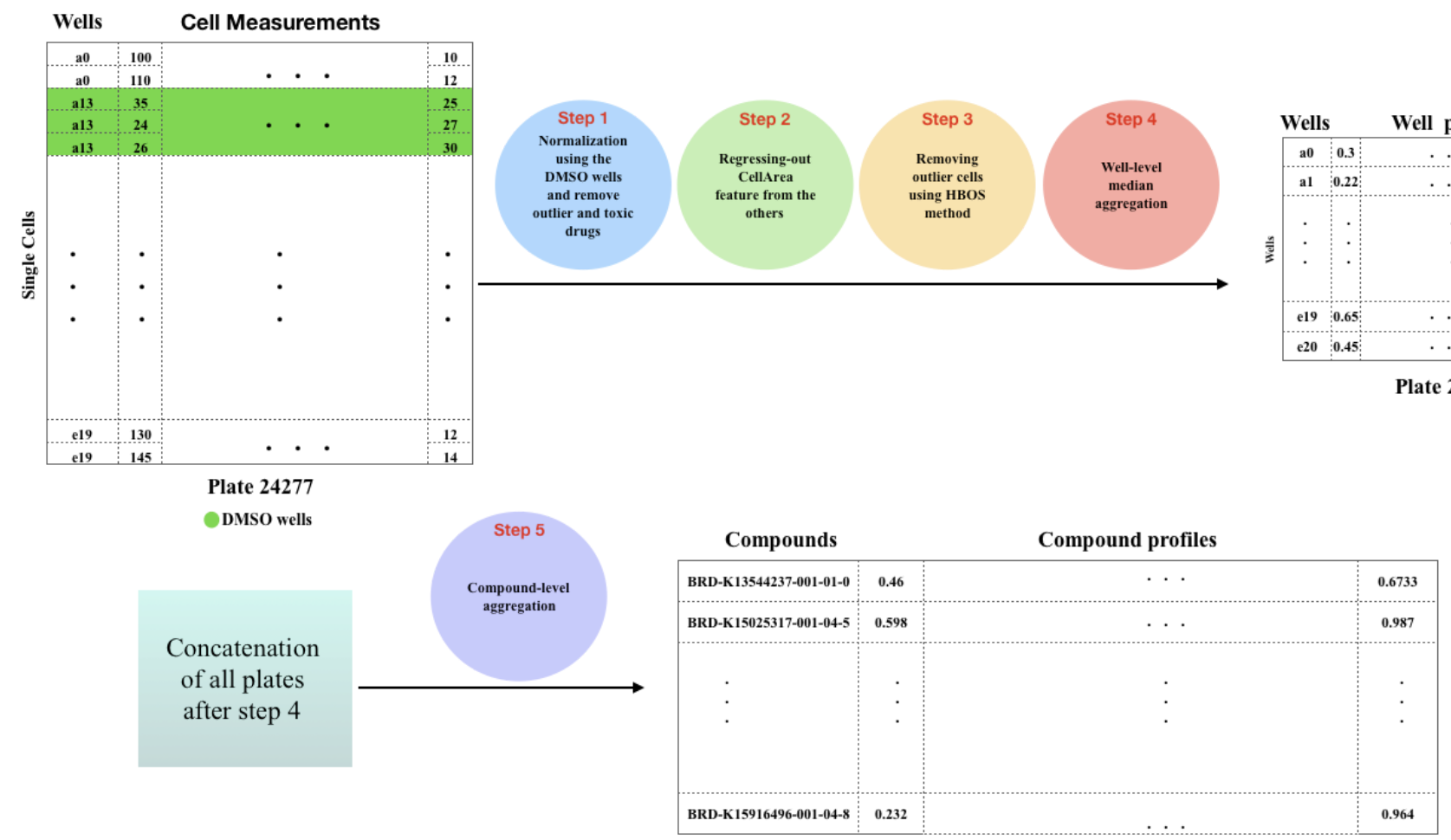

Fig 1. Representative workflow for the enhanced image-based cell profiling via data cleaning. Five main steps transform cell measurements of each plate to compound-level profiles. 


\section{Experiments and results}

\subsection{Data cleaning}

The enrichment of $k$ percentages of compound pairs with highest profile correlation in having the same MoA is used for the evaluation. To measure the enrichment, the ratio of pairs with the same MoA to different MoAs is calculated, and compared to the same ratio for weakly correlated pairs. This comparison could be summarized into a single number known as the odds ratio, which is described in the Material and Methods. This basically shows how many times we expect to find biologically related compound pairs in the top $k$ percent connections compared to the other connections.

We first investigated a certain feature transformation step that aims at removing the cell area information that is implicitly contained in other features. This is particularly useful given that many morphological features, such as the perimeter, or texture, are affected by the cell area. We simply form a linear regression to predict any given feature from the cell area at the single-cell level. The residual, or error, of such a regression represents the information that is uncorrelated to the cell area. This process, which we call "regressing out," helps in balancing the effect of cell area in the profiles. Fig. 2 left, shows that the odds ratio has improved slightly as a result of this step. We make comparisons in two cases. In the first case, all compounds in the dataset are included, while in the second case, only the compounds with a high replicate correlation are retained. This filtering has previously been discussed to be a key step in removing treatments that do not show any strong biological effect from the lens of the image-based assay (4). We call such compounds "outlier" throughout the paper. It is notable that the odds ratio jumps to a much higher level, from $\sim 9$ to higher than 40 , when such compounds are removed. In this case, regressing cell area out results in a more consistent improvement in the odds ratio across various values of $k$, which is illustrated in Fig. 2 right. 
We next investigated the effect of removing overly toxic compounds, which highly affect the cell viability. We would expect a much smaller cell count in the wells that are treated by such compounds. The profile might be of lower reliability as a result of the small cell count. In addition, the profiles of toxic compounds are so unique that artificially inflates the odds ratio. The increase in the odds ratio as a result of toxicity is not valuable from a practical perspective, due to limited clinical use of overly toxic compounds, and in drug discovery as a result. Therefore, we opted to remove such compounds before making further evaluations. Fig. 3 left, shows that regress-out gives a more pronounced improvement over the odds ratio when both outlier and toxic compounds are removed. This could be partly due to regress-out unreliability under small sample size, which is the cell count in this case.

Finally, we applied outlier detection at the level of single-cells through a histogram-based technique, which is called "hbos." Building upon our previous improvements, we further removed outlier cells prior to making the profiles. The odds ratio keeps enhancing in all the thresholds, as shown in Fig. 3 right. It turns out that the cell outlier detection is a more effective method in improving the overall profile quality, while regress-out mainly improves the very top connections.

To assess the combined effectiveness of regress-out and cell outlier removal, we take as baseline the non-toxic and inlier drugs in addition to one of these methods, and apply the other method next. Regress-out seems to provide marginal additional improvement over cell outlier removal, Fig. 4 left, while cell outlier detection gives more extra enhancement over regress-out as shown in Fig. 4 right. We conclude that both of these methods are valuable in cleaning the profile, and provide independent improvements, but the cell outlier removal is a bit more effective. All techniques are described in more detail in the Material and Methods section. 

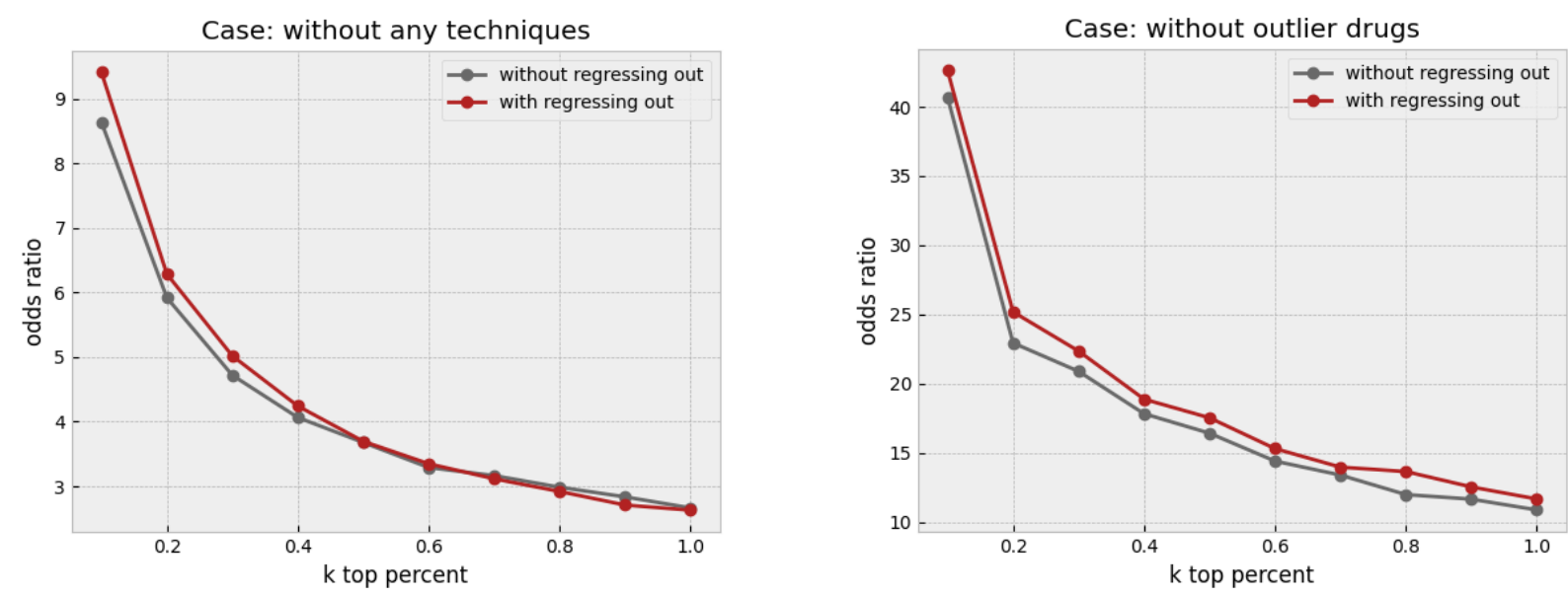

Fig 2. Effect of regressing-out the cell-area. The left plot shows the increase in the odds ratio by regressing out the cell area from all other features in different $\mathrm{k}$ percentages when no other data cleaning method is applied. The right plot shows that regressing out improves the result when just outlier drugs, which exhibit weak phenotypes, were removed.
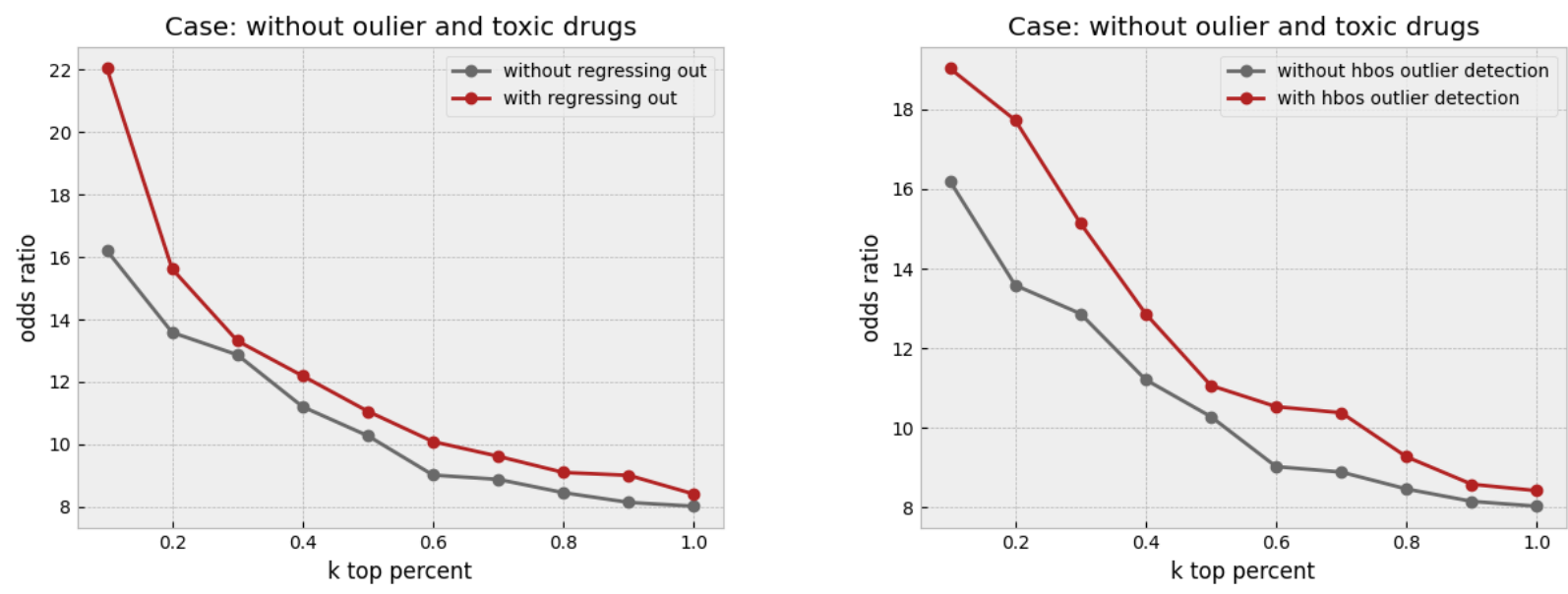

Fig 3. Effect of regressing-out and cell outlier detection. The left plot shows the increase in the odds ratio by regressing out the cell area from all other features in different $\mathrm{k}$ percentages when outlier and toxic drugs were removed. Improvement in the odds ratio becomes more significant when the toxic drugs are excluded from the analysis. The right plot shows that using histogram-based outlier detection improves the result in the same baseline case, where the outlier and toxic drugs were removed. 

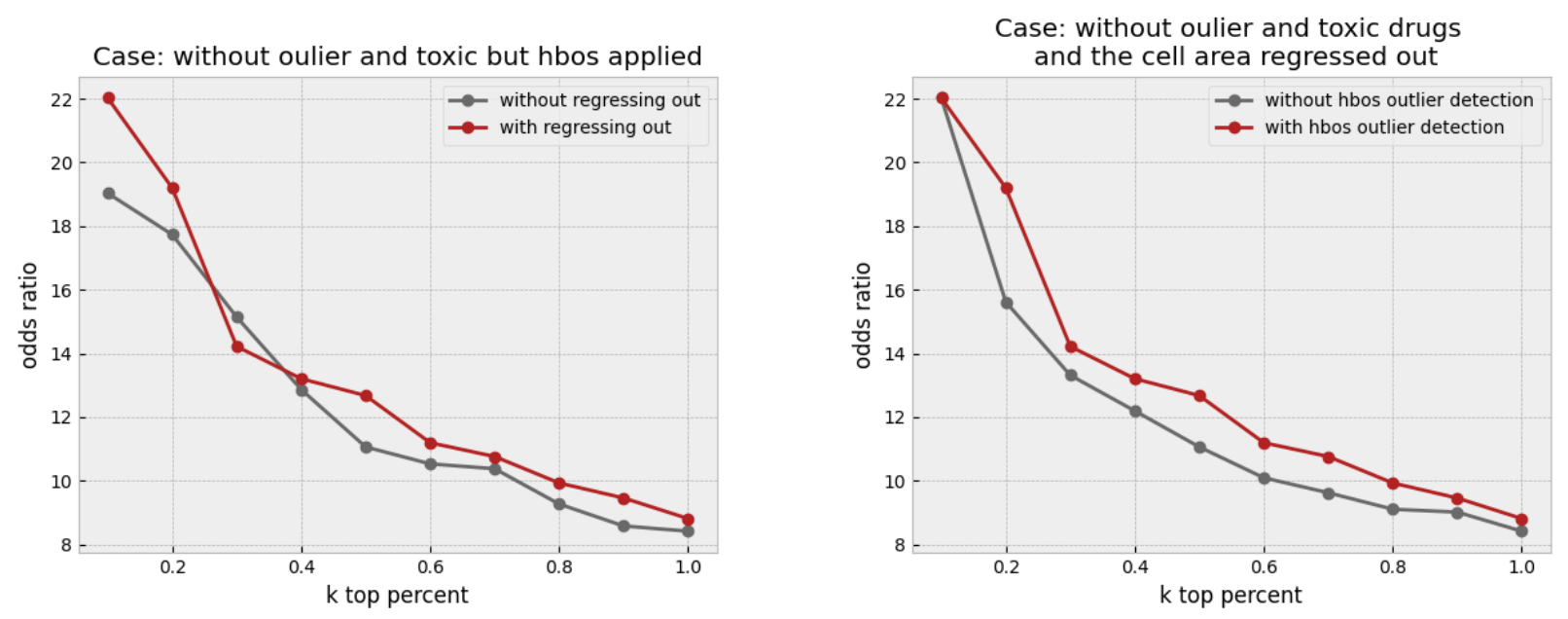

Fig 4. Combined effect of outlier detection and regressing-out. The left plot shows the effect of regressing out when all other preprocessing steps (outlier and toxic drug removal, and cell outlier removal) are applied. The right plot shows the effect of cell outlier removal when all other steps, including outlier and toxic drug removal and regress-out, have been applied. Cell outlier removal seems to provide a more consistent improvement across values of $k$ compared to the regress-out technique.

\subsection{Analysis of deep learning methods}

Unfortunately, none of the deep representation learning methods, which are described in Material and Methods, could lead to a better result in the odds ratio (Supp. Fig. 14 and 15). There are some explanations for these observations. The most important factor that prevents representation learning, could be information loss, which is a result of using CellProfiler output. In fact, there are some important features that are captured using CellProfiler, but they are mostly the features that are introduced as important features by biologists, and there could be some hidden patterns in the raw images that a deep neural network is capable of learning, which are not captured by CellProfiler. For example, the texture information in the CellProfiler features are limited to certain Haralick texture descriptors, while a deep convolutional model could learn richer texture patterns. One other source of information loss in this work, is that the raw images contain 16 bits of 
information for each pixel. This means that they can be modeled more accurately when fed originally to a deep model, compared to the CellProfiler features, which are often of lower precision.

Another reason that the network does not improve the results is that there are some problems with outputs of CellProfiler for some images with low quality or with a small number of cells in them. This problem was explained in the previous sections. All these observations and hypotheses lead to one conclusion, that it is challenging for the neural networks to improve the CellProfiler output, and they should be trained on the raw images instead to be able to capture the high-quality information.

\section{Material and method}

\subsection{Dataset}

We used a dataset of images and morphological profiles of small-molecule treatments using the Cell Painting assay (6). About 50 plates, which correspond to the bioactive compounds with known mechanisms of actions, are chosen. Considering redundant features extracted by CellProfiler, a specific list of features are selected for this experiment. This feature and plate selection is based on the method proposed by Rohban et al. (4) . Each plate consists of wells that consist of cells and each well is treated by a particular compound. There are wells known as negative controls (DMSO), which are treated only with the compound solvent. The profile of a well is obtained by taking the median of single-cell measurements in that well. To mitigate the batch effect, profiles in each plate were normalized separately using the DMSO wells of that plate. For normalizing cells of a plate, single cells were subtracted by the median of DMSO well profiles and divided by the median absolute deviation (MAD) of DMSO well profile (3). There are several strategies for creating 
aggregated profiles. In this work, similar to earlier experiments $(3,4)$, the median and profiles are used for well-level and profile-level aggregations.

\subsection{Replicate correlation}

Not all of the compounds produce a meaningful change in the features extracted by CellProfiler. Therefore, a drug selection method must be used, to only keep those drugs with discernible effects on the features (7).

First, a median profile for each well is calculated. Then, the wells are grouped based on their "Metadata_broad_sample" column, so each group contains profiles of a specific compound. The similarity measure is chosen to be Pearson's correlation, which reflects the linear relationship between two variables. The more similar the profiles are between two wells, the higher the correlation coefficient will be. To select drugs with meaningful effect on the features, a null distribution is required. The null distribution is defined based on the correlations between non-replicates. That is, the median correlation between groups of $k$ different compounds constitute samples of the null distribution, where $k$ is the number of technical replicates in the experiment. Compounds whose median replicate correlations are greater than the 95th percentile of the null distribution are selected for further steps (7) (8), as they have a meaningful effect on the cells. We will refer to the drugs removed in this section as the "outlier drugs" in the following sections. In this experiment, there were a total of 1551 different compounds, and based on the explained method, only 455 of them were kept.

\subsection{Odds ratio}

Since similarity metrics reveal connections among image-based profiles, choosing a suitable one can enhance the analysis. Like similar tasks, Pearson's correlation coefficient was used in this work. 
Consider two wells, each treated by a compound, which has some special mechanism of action, or "MoA" for short. In this experiment, the final purpose is to find whether there is any association between high values of correlations of the profiles of two wells and similarity of the MoA of compounds that are used to treat the cells in those wells. In other words, it is hypothesized that if profiles of two wells have a high correlation, they are probably treated with compounds of the same MoA. To test this hypothesis, one-sided Fisher's exact test is used.

If the Fisher's test admits the hypothesis, this process can be used for drug discovery, as it is possible to do high throughput experiments and use the correlation of profiles to guess MoA of unknown compounds.

In this experiment, odds ratio (9) is used to check how likely it is that the hypothesis is true. The Fisher's test contingency table that is used in our experiment can be found in Table 1.

Table 1. Odds ratio: Odds ratio is calculated from the contingency table as $(\mathrm{a} / \mathrm{b}) /(\mathrm{c} / \mathrm{d})$.

\begin{tabular}{|c|c|c|}
\hline & Same MoA & Different MoAs \\
\hline Compound pairs with & $\mathrm{a}$ & $\mathrm{c}$ \\
highest correlation $(\boldsymbol{k}$ & & \\
percent of all pairs) & & $\mathrm{d}$ \\
\hline Other pairs & $\mathrm{b}$ & \\
\hline
\end{tabular}


We change the value of " $k$ " in table 1 to draw a plot, where its $y$ axis corresponds to the odds ratio value, and its $x$ axis corresponds to value " $k$ ", or the percentage of pairs with highest correlations.

If the hypothesis is true, we expect to have a plot with a negative slope. That means that by tightening the condition of correlation, there must be more similarities in MoAs.

\subsection{Regressing-out}

The majority of features are affected by a feature named Cells_AreaShape_Area. This feature expresses the space that is occupied by each cell. We used the regress-out technique to reduce the impact of this feature on the rest in order to strengthen the meaning of each feature individually. For this purpose, the relation between each feature and Cells_AreaShape_Area of all cells in one plate was modeled by fitting a linear equation without intercept.

Considering $x$ as Cells_AreaShape_Area feature of all single-cells in a plate and $y$ as another feature from the feature set of all cells. In another word, $x_{i}$ represents the area and $y_{i}$ another feature of the $i-t h$ cell in that plate. A linear regression line without intercept attempts to fit a line that has an equation of the form $y=a x$.

After fitting the model for each feature and cell area, that feature column was replaced by their residuals from the regression prediction. Residual in regression means the difference between any data point and the regression line:

$$
\text { Residual }=\text { real value }- \text { predicted value }
$$

For each feature except cell area and for each plate, this technique and replacement have to be done separately. By using this technique, significant progress was observed in the final results and odds ratio in different first $k$ percentages. 


\subsection{Histogram-based outlier score}

By investigating images of some wells and the histogram of multiple features, it was crystal clear that there are some outlier profiles. The cause of this could be the issues in the pipeline or the imaging process. There are several ways for detecting outliers in a dataset. Three different setups for outlier detection are supervised, semi-supervised, and unsupervised methods. For the first two setups, labeled data is needed. In this dataset, outlier cells are not labeled, therefore unsupervised methods have to be adopted.

There are various approaches for unsupervised outlier/anomaly detection. Two well-known methods are Local Outlier Factor (LOF) (10), nearest-neighbor-based algorithm (11), and Clustering Based Local Outlier Factor (CBLOF) (12), a clustering-based algorithm. Both are computationally expensive. A fast and efficient unsupervised method for outlier detection is the Histogram-Based approach (13). This approach assumes independence of features, so outlier detection is most appropriately applied after regressing out, where the features become more independent.

Histogram-Based Outlier Score for an instance is calculated by building a histogram for each feature and aggregating them. The proportion of outliers or the amount of contamination of the dataset is a hyperparameter. In this task, for each plate containing wells, histogram-based outlier detection is applied separately with a 0.01 amount of contamination, and the outlier cells are removed. A useful library in Python for this section is PyOD (14).

\subsection{Toxic drugs}

While investigating the features of each well, we faced some wells with an extraordinarily low number of cells, even less than 10 cells in a whole well, as shown in Fig. 5. 


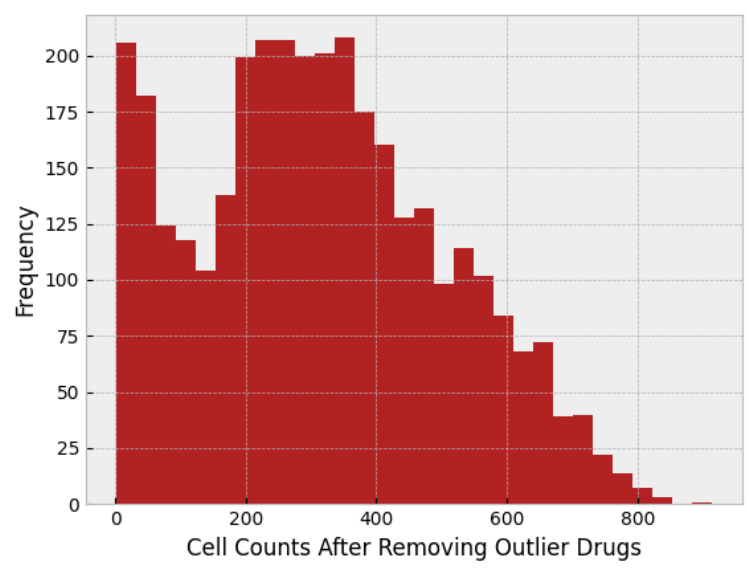

Fig 5. Histogram of the cell count in each well

This issue could have several sources. It could be the result of a bad vital situation such as lack of nutrients in a well, that caused the cell death. Or, it could be the result of the drug itself. Some drugs have a toxic nature, and cause cell death.

If the drug is a toxic one, that may cause a false result in the final odds ratio plot. To find toxic drugs, we checked the median of cell count of different wells facing the same drug, and we removed 0.05 of the drugs with the lowest cell count median. Cell count histogram after removing toxic drugs can be found in Fig. 6.

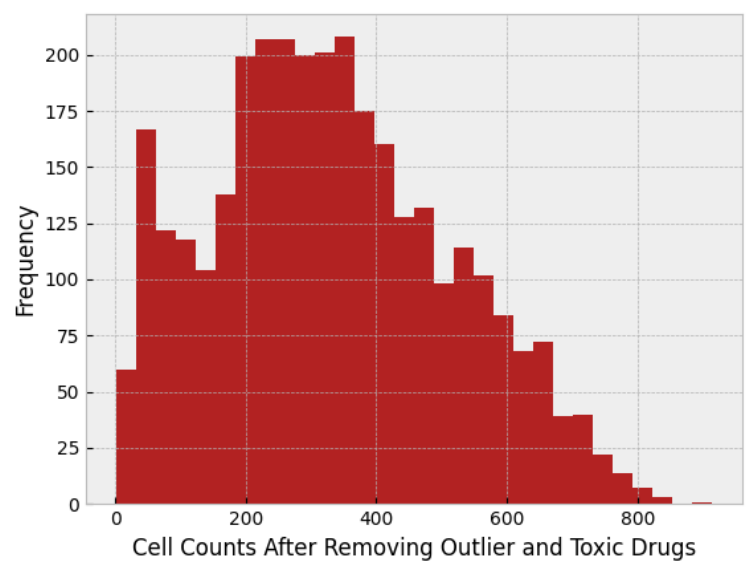


Fig 6. Histogram of the cell count after removing toxic drugs

After doing so, the odds ratio was decreased. This indicates that the toxic drugs have a correlation in their features that by removing them from the experiment, odds ratio decreases, and toxic drug removal makes the final result more real. Fig 7. shows the correlation between cells affected by toxic drugs, and Fig 8. corresponds to correlation between toxic and non-toxic drugs. The comparison between these figures makes it clear that toxic drugs are highly correlated.

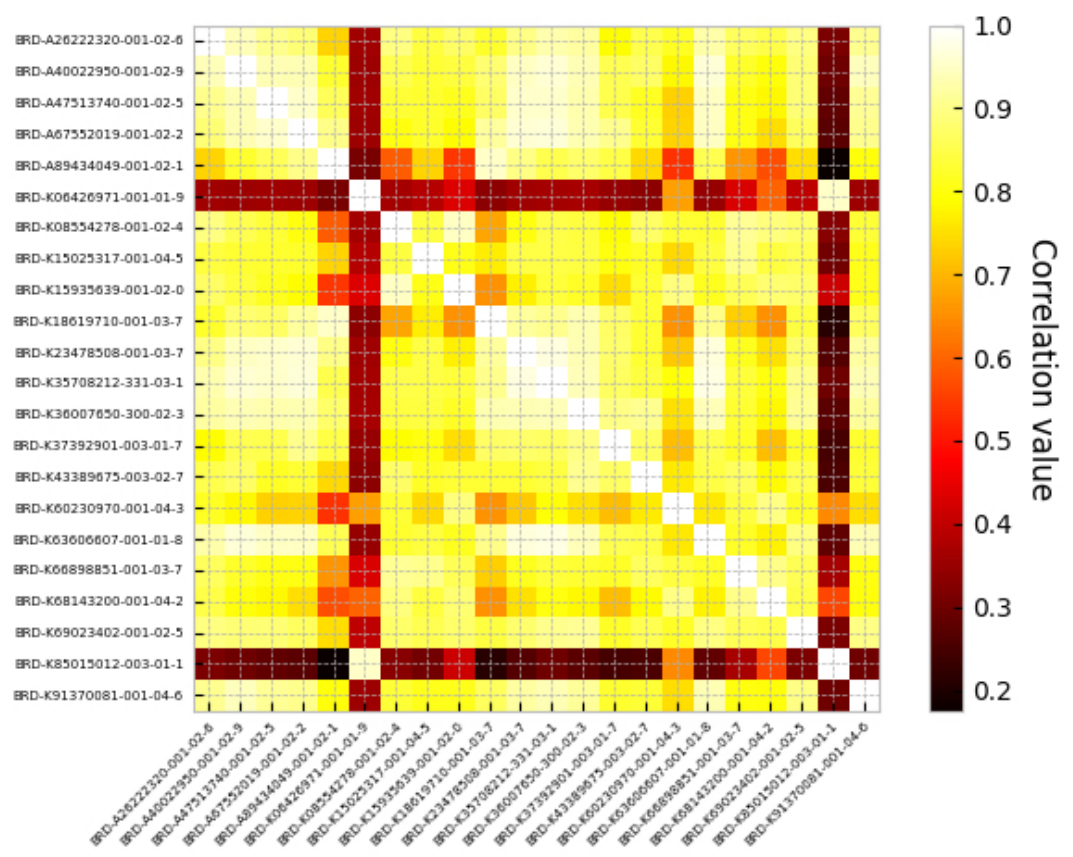

Fig 7. Correlation between toxic drugs

Fig 8. Correlation between toxic drugs and non-toxic drugs. Toxic drugs are in rows and non-toxic ones are in columns. 
Regress-out and outlier detection using HBOS both improved the odds ratio after removing toxic drugs, which confirms our previous results.

\subsection{Deep learning}

Recently, deep neural networks have appeared very promising in representation learning on various problems. Several methods were studied for the purpose of learning more meaningful representations to increase the similarity between drug embeddings with the same mechanism of action. Both supervised and unsupervised approaches are tested and a brief explanation for each of them is discussed next.

\subsubsection{Classification with HSIC loss}

A simple model is designed for this task. The input of the network is single-cell features after feature selection and normalization, which was previously explained, and the output that the model has to predict is the drug by which that cell in the input is treated with. We expect the network to learn a useful representation of cells through this auxiliary task of predicting drug identity from the cellular measurements. For preparing the training data, five mechanisms of action are chosen and about 30 drugs with these MoAs are selected randomly. All cells in various pairs of plates and wells that are treated by these 30 compounds are collected to form the training data. An important point to be careful in the train-test split is to not have cells from the same well/drug in both train and test/validation data.

The purpose of this random selection of compounds and not to use all of them in training and designing a network is to assess reasonable generalization on unseen data, especially new compounds whose mechanisms and similarities with others are still unknown. Let $(X, y)$ be a data point such that $X \in R^{470}$ and 
$y \in R^{30}$. As discussed earlier, each single-cell profile has about 470 features (less than 470), which are extracted by CellProfiler, and the output is probabilities for each of 30 compounds.

The loss function that is used for this task is Hilbert-Schmidt Independence Criterion (HSIC) loss (15) with the aim of learning on the training data with a limited number of MoAs and compounds but generalize and perform well on profiles that have not been seen by the model. This loss function has recently been proposed to facilitate out-of-distribution generalization. Few changes in the implementation of HSIC loss by authors are needed to use it for the multi-class classification.

The model is constructed by three dense layers. The activation function that is used for all layers is ReLU and after the last layer softmax was applied to the outputs. After training and convergence of loss on the train data, the trained model can be used for extracting new representations for every single cell. The second last layer or the hidden layer before the class probabilities (the red layer shown in Fig 9.) is our new representation for the input single-cell profile.

After finding the representation of every single cell by passing them through the trained model, all previous steps such as aggregation, calculating the correlation between the compounds' profile, and plotting are repeated. The results from the new representations were meaningless, and were not promising compared to raw profiles, and did not improve the performance. 


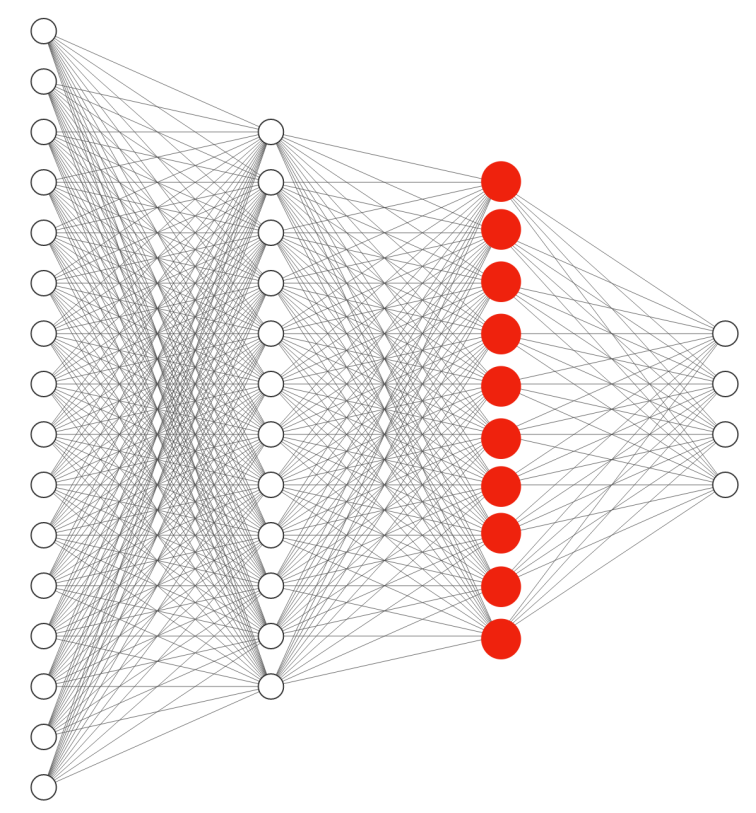

Fig 9. Simple classification network architecture. New representation from the classification task to feed into the pipeline is created by passing through the trained network and picking the red layer.

\subsubsection{Autoencoders}

Recently, Autoencoder (16) has achieved remarkable success as a feature extraction method. A basic autoencoder has two parts, an encoder, and a decoder. The output of the encoder represents the reduced representation of input data (code) and the decoder reconstructs the original input from the latent code that is generated by the encoder. Later, other variations of autoencoders were proposed that each tried to solve an issue such as variational autoencoder (VAE) (17), denoising autoencoder (DAE) (18), sparse autoencoder (SAE) (19), relational autoencoder (20), and etc. In this section, preparing the training data is quite similar to the classification task. Since this method is unsupervised, labels (compounds' names) are not needed. The decoder of the model should reconstruct the input of the encoder, which is a single-cell profile. After training and convergence of the mean squared loss on the training data, the trained model can be used for extracting 
new representations for every single cell. The code layer or the output of the encoder (the red layer shown in Fig. 10) is the new representation for the input single-cell profile from the autoencoder.

After finding the representation of every single cell by passing them through the trained model, all previous steps for calculating the odds ratio are repeated. Basic AE, VAE, SAE, RAE, and DAE with different depths and code sizes were tested but no significant improvement was observed in the final results.

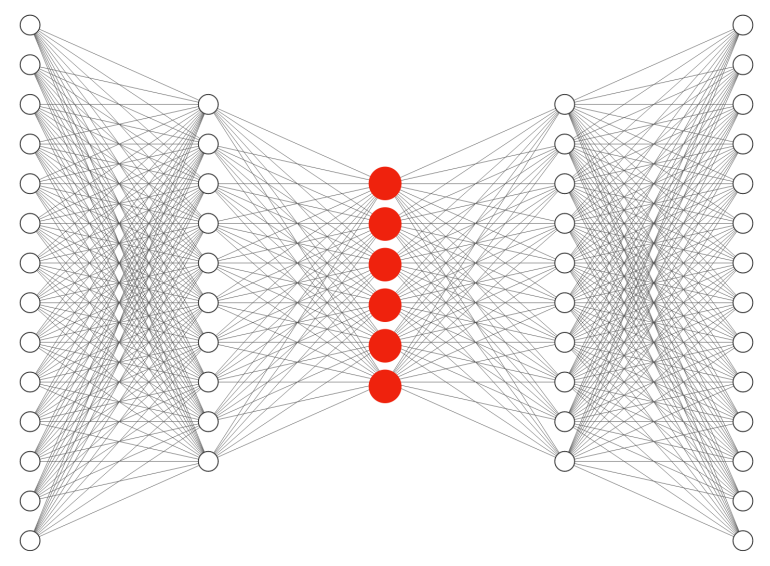

Fig 10. Autoencoder network architecture. New representation from autoencoders to feed into the pipeline is created by passing through the trained network and picking the red layer.

\subsubsection{Mixup}

According to (21), one way to improve model generalization is to construct some virtual training data:

$$
\begin{array}{ll}
\hat{x}=\lambda x_{i}+(1-\lambda) x_{j,} & \text { where } x_{i}, x_{j} \text { are raw input vectors } \\
\hat{y}=\lambda y_{i}+(1-\lambda) y_{j,} & \text { where } y_{i}, y_{j} \text { are one-hot label encodings. }
\end{array}
$$

As proposed in the paper, mix-up is based on the prior knowledge that linear interpolations of feature vectors should lead to linear interpolations of the associated targets. This technique is used in the 
classification steps that were covered earlier in the HSIC section. It led to better accuracy on the test set of the auxiliary task, but no significant improvement in the odds ratio was observed.

\subsection{Images}

After feeding CellProfiler outputs to deep neural networks and getting poor results, we investigated some of the images to check if any problems with them cause errors somewhere in the CellProfiler pipeline.

Some of the wells that were containing DMSO had some intensity issues. This may mislead CellProfiler algorithms and cause them to extract noisy information. Noisy DMSO can affect all the information of a plate, as it is used for normalization.

In Figs 11 and 12, images of some misleading DMSO wells can be seen.
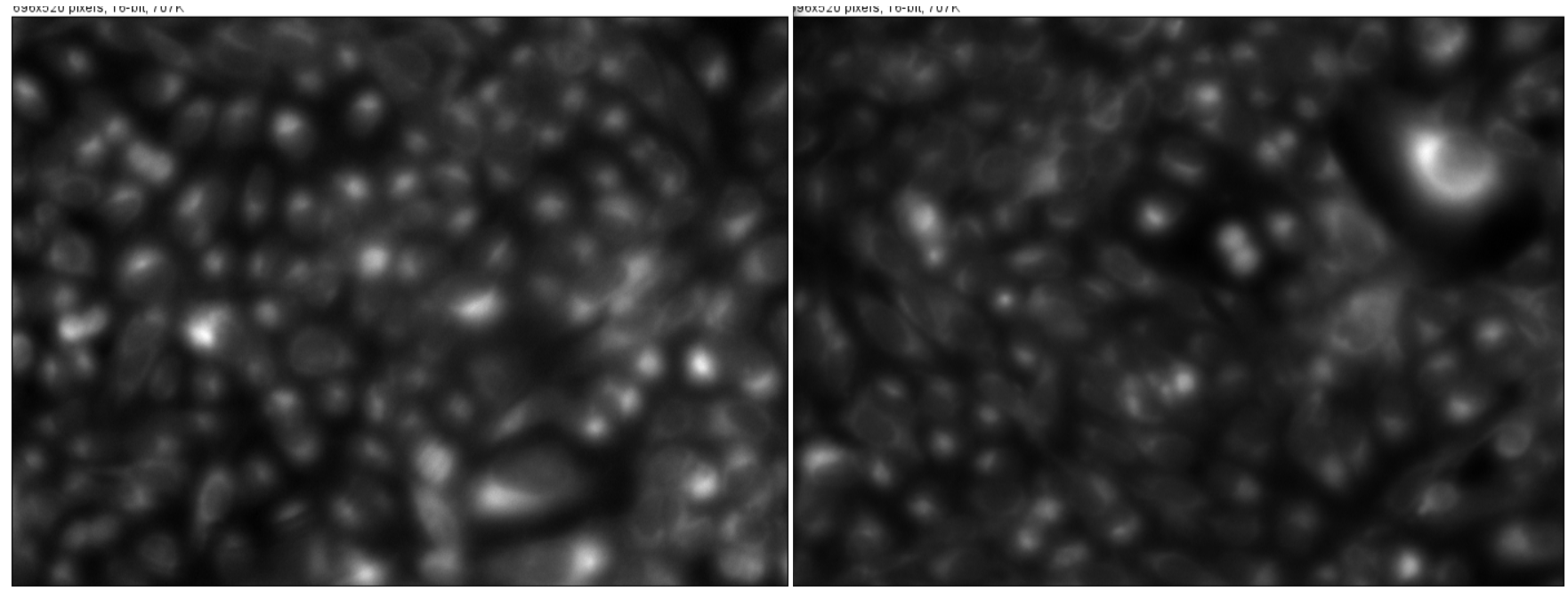

Fig 11. Plate 24277, well A13, containing DMSO, is blurry 

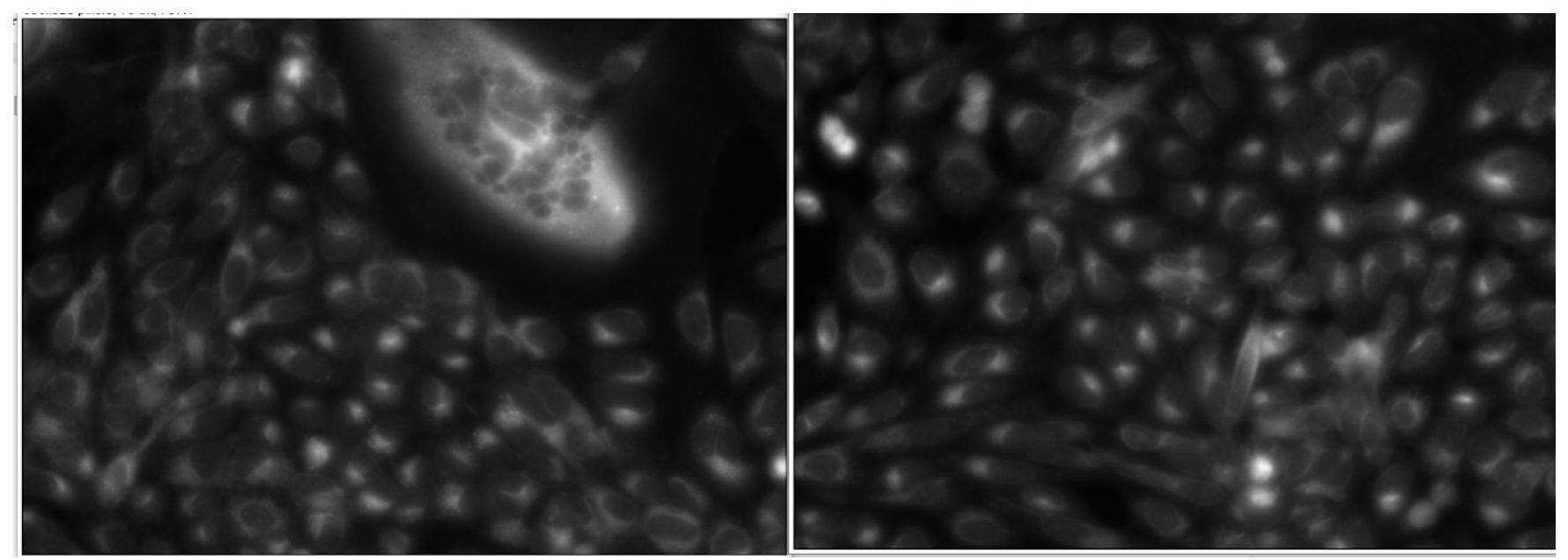

Fig 12. Another outlier DMSO well in plate 24293

Some wells had an extraordinary high cell area. By investigating their raw images it was clear that it is CellProfiler's mistake. Those images were mostly toxic wells containing a very small number of cells. For example, plate 24293, well E19, has a cell area of nearly 3000 after normalization (Fig. 13).

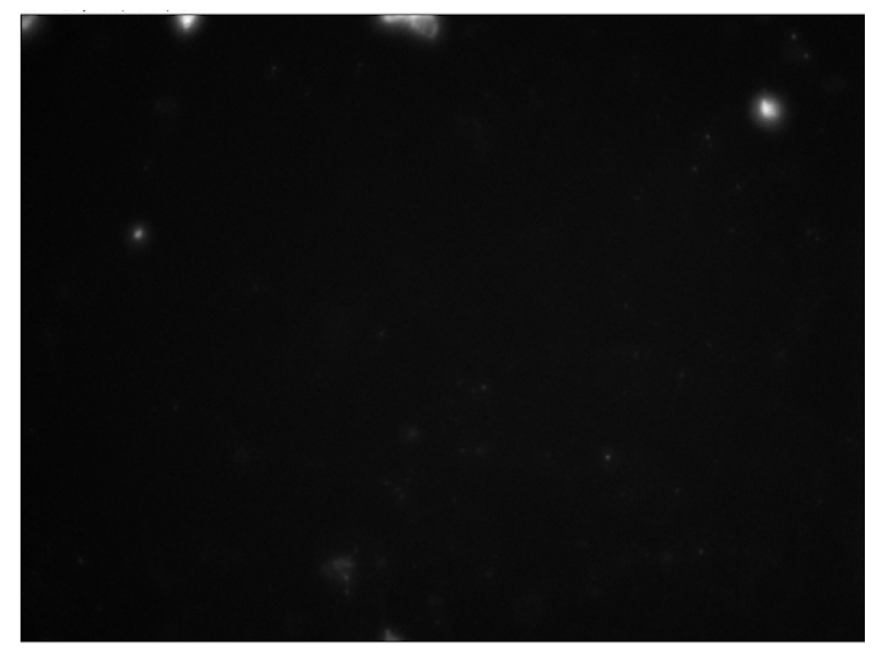

Fig 13. plate 24293, well E19, with a cell area of nearly 3000

As mentioned earlier, the noise in the images, especially in DMSO wells, could be a source of error in the CellProfiler pipeline, and this indicated the importance of data cleaning in image-based profiling enhancement. 


\section{Conclusion}

High throughput assays play a vital role in carrying out biomedical experiments. CellProfiler is a common tool that is used to quantify the data, which is produced by these assays, but many sources of error might affect the data quality in this pipeline. In this work, we mainly focused on data cleaning steps that could enhance image-based profiles extracted from CellProfiler.

Our Experiments indicate that data cleaning methods highly impact the quality of extracted features to identify mechanisms of action of different drugs, which results in a higher amount of the odds ratio. Removing drugs with intra-correlation less than inter-correlation improves the odds ratio. It is the result of keeping those drugs with more meaningful features. Removing toxic drugs - drugs that cause cell death decreases the odds ratio, but makes the result more real and meaningful.

Due to the different error sources in the imaging and feature extracting pipeline, cell level outlier detection is an important step to enhance the profile of each specific compound. HBOS outlier detection was used in this work. Regressing out the cell area from all other features is another helpful step in data cleaning, since cell area widely affects all other features, and by regressing it out, other useful information can be presented better in the resulting profile. Both techniques proved to be effectively improving the odds ratio.

Deep representation learning methods that are applied on top of CellProfiler features could not achieve a result better than the raw features, mainly because of information loss in the CellProfiler measurement step, but they have a perfect ability in capturing hidden patterns, and they may be useful if applied on the raw images instead. 


\section{Code availability}

All code for data cleaning and analysis associated with the current submission is available at https://github.com/rohban-lab/Data-Cleaning-for-image-based-profiling-Enhancement.

\section{Data availability}

The dataset used in this work is CDRPBIO-BBBC036-Bray. This dataset is the bioactive subset of a publicly available dataset (6). Image-based profiles are available at http://gigadb.org/dataset/view/id/100351.

\section{Author contributions}

Arghavan Rezvani \& Mahtab Bigverdi: Formal Analysis; Methodology; Software; Investigation; Visualization; Writing - Original Draft Preparation; Writing - Review \& Editing; Visualization;

Mohammad Hossein Rohban: Supervision; Conceptualization; Resources; Project Administration; Investigation; Validation; Writing - Review \& Editing.

\section{References}

1. Pegoraro G, Misteli T. High-Throughput Imaging for the Discovery of Cellular Mechanisms of Disease. Trends Genet TIG. 2017 Sep;33(9):604-15.

2. Caicedo JC, Cooper S, Heigwer F, Warchal S, Qiu P, Molnar C, et al. Data-analysis strategies for image-based cell profiling. Nat Methods. 2017 Sep;14(9):849-63.

3. Ljosa V, Caie PD, ter Horst R, Sokolnicki KL, Jenkins EL, Daya S, et al. Comparison of Methods for Image-Based Profiling of Cellular Morphological Responses to Small-Molecule Treatment. J Biomol Screen. 2013 Dec;18(10):1321-9.

4. Rohban MH, Abbasi HS, Singh S, Carpenter AE. Capturing single-cell heterogeneity via data fusion improves image-based profiling. Nat Commun. 2019 Dec;10(1):2082.

5. Carpenter AE, Jones TR, Lamprecht MR, Clarke C, Kang IH, Friman O, et al. CellProfiler: image analysis software for identifying and quantifying cell phenotypes. Genome Biol. 2006 Oct 31;7(10):R100. 
6. Bray M-A, Gustafsdottir SM, Rohban MH, Singh S, Ljosa V, Sokolnicki KL, et al. A dataset of images and morphological profiles of 30000 small-molecule treatments using the Cell Painting assay. GigaScience [Internet]. 2017 Dec 1 [cited 2021 May 30];6(12). Available from: https://academic.oup.com/gigascience/article/doi/10.1093/gigascience/giw014/2865213

7. Rohban MH, Singh S, Wu X, Berthet JB, Bray M-A, Shrestha Y, et al. Systematic morphological profiling of human gene and allele function via Cell Painting. Settleman J, editor. eLife. 2017 Mar 18;6:e24060.

8. Koch CM, Chiu SF, Akbarpour M, Bharat A, Ridge KM, Bartom ET, et al. A Beginner's Guide to Analysis of RNA Sequencing Data. Am J Respir Cell Mol Biol. 2018 Aug;59(2):145-57.

9. Szumilas M. Explaining Odds Ratios. J Can Acad Child Adolesc Psychiatry. 2010 Aug;19(3):227-9.

10. Breunig MM, Kriegel H-P, Ng RT, Sander J. LOF: Identifying Density-Based Local Outliers. :12.

11. Ramaswamy S, Rastogi R, Shim K. Efficient Algorithms for Mining Outliers from Large Data Sets. :12.

12. He Z, Xu X, Deng S. Discovering cluster-based local outliers. Pattern Recognit Lett. 2003 Jun 1;24(9):1641-50.

13. Goldstein M, Dengel A. Histogram-based Outlier Score (HBOS): A fast Unsupervised Anomaly Detection Algorithm. :1.

14. Zhao Y, Nasrullah Z, Li Z. PyOD: A Python Toolbox for Scalable Outlier Detection. :7.

15. Greenfeld D, Shalit U. Robust Learning with the Hilbert-Schmidt Independence Criterion. ArXiv191000270 Cs Stat [Internet]. 2020 Jul 11 [cited 2021 May 30]; Available from: http://arxiv.org/abs/1910.00270

16. Rumelhart DE, Hinton GE, Williams RJ. Learning representations by back-propagating errors. Nature. 1986 Oct;323(6088):533-6.

17. Goodfellow I, Pouget-Abadie J, Mirza M, Xu B, Warde-Farley D, Ozair S, et al. Generative Adversarial Nets. In: Ghahramani Z, Welling M, Cortes C, Lawrence N, Weinberger KQ, editors. Advances in Neural Information Processing Systems [Internet]. Curran Associates, Inc.; 2014. Available from: https://proceedings.neurips.cc/paper/2014/file/5ca3e9b122f61f8f06494c97b1afccf3-Paper.pdf

18. Vincent P, Larochelle H, Bengio Y, Manzagol P-A. Extracting and composing robust features with denoising autoencoders. In: Proceedings of the 25th international conference on Machine learning ICML '08 [Internet]. Helsinki, Finland: ACM Press; 2008 [cited 2021 May 30]. p. 1096-103. Available from: http://portal.acm.org/citation.cfm?doid=1390156.1390294

19. Deng J, Zhang Z, Marchi E, Schuller B. Sparse Autoencoder-Based Feature Transfer Learning for Speech Emotion Recognition. In: 2013 Humaine Association Conference on Affective Computing and Intelligent Interaction. 2013. p. 511-6.

20. Meng Q, Catchpoole D, Skillicom D, Kennedy PJ. Relational autoencoder for feature extraction. In: 2017 International Joint Conference on Neural Networks (IJCNN). 2017. p. 364-71.

21. Zhang H, Cisse M, Dauphin YN, Lopez-Paz D. mixup: Beyond Empirical Risk Minimization. In 2018 [cited 2021 May 30]. Available from: https://openreview.net/forum?id=r1Ddp1-Rb 


\section{Supplementary Materials}

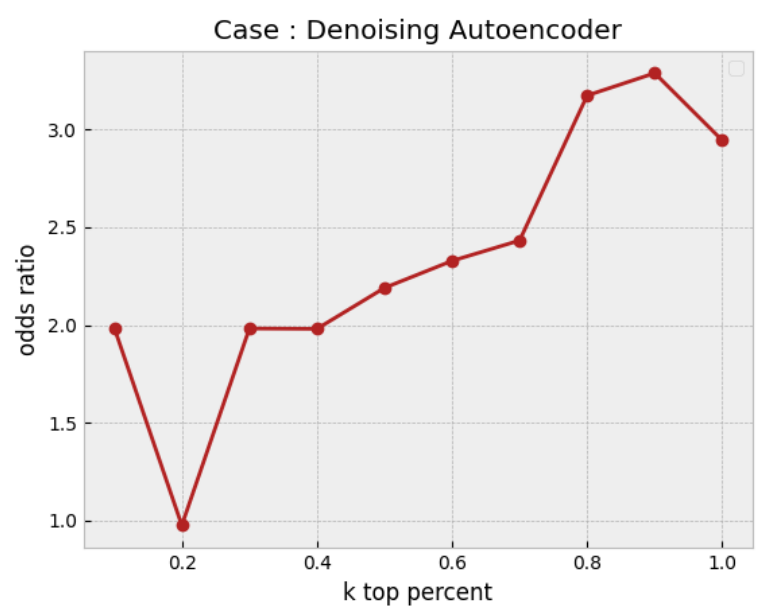

Fig 14. Odds ratio for profiles that are obtained through representation learning using a denoising autoencoder. A denoising autoencoder (DAE) with one hidden layer of encoder and decoder, and code size of 200 is trained. Compound-level profiles that are used for the last step come from the aggregation of DAE representation of cell measurements. Odds ratios in different percentages are too low and full of fluctuations compared to the cases that were investigated in the results section.

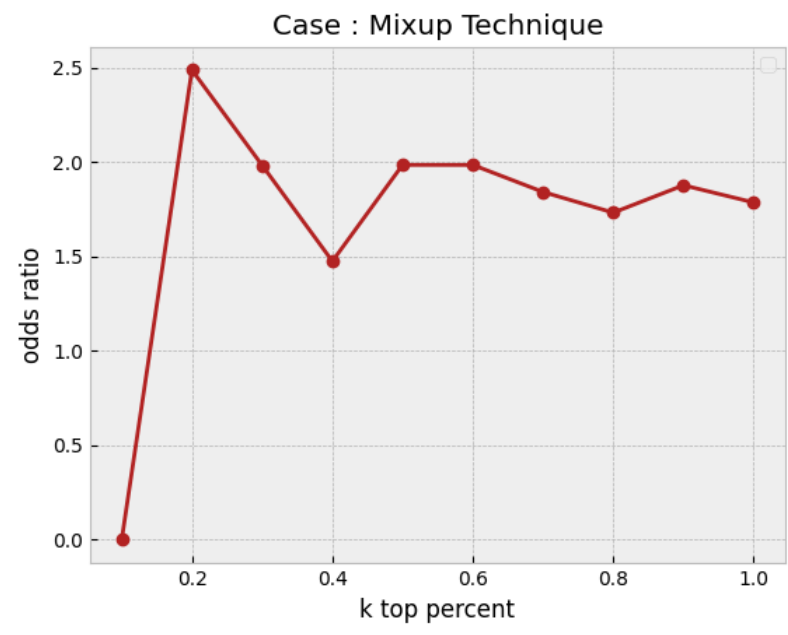


bioRxiv preprint doi: https://doi.org/10.1101/2021.09.09.459624; this version posted September 10, 2021. The copyright holder for this preprint (which was not certified by peer review) is the author/funder. All rights reserved. No reuse allowed without permission.

Fig 15. Odds ratio for profiles that are obtained through a supervised representation learning using the mixup technique. A simple fully connected network that is regularized through applying the mixup technique is trained. In the first step, instead of raw cell-level profiles, representations that are extracted from the network are used. Considering this plot, the mixup technique does not effectively improve upon the baseline that was discussed earlier in the results section. 